\title{
Editorial
}

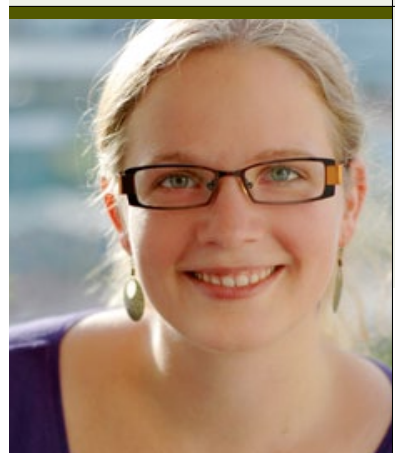

"Es müssen mehr Anreize geschaffen werden, sektorenübergreifende Behandlungskonzepte im Versorgungsalltag umzusetzen - zum Wohle unserer Patienten."

\section{Sektorenübergreifende Betreuung von Schmerzpatienten lohnt sich}

$$
\text { C }
$$
hronische Schmerzen gehen häufig mit erheblichen körperlichen, psychischen und sozialen Belastungen einher. Oft suchen Betroffene bei vielen unterschiedlichen Ärzten Hilfe für ihr Schmerzproblem. Dieses sog. „doctor hopping“" verursacht nicht nur immense Kosten, es führt auch zu fragmentierten Patientenkarrieren. Diese sind geprägt durch eine Vielzahl unimodaler Behandlungsversuche, die angesichts der Komplexität und Langwierigkeit chronischer Schmerzen zu einer Über-, Unter- oder Fehlversorgung führen können. Daher erfordern Patientenbehandlungen ohne Brüche ein hohes Maß an inter- und intrasektoraler Vernetzung. Verschiedene Leistungserbringer entwickelten hierzu innovative Konzepte [Schmerz. 2013;27(2)].

Der Goldstandard der Therapie von Patienten, die an einem chronischen Schmerzsyndrom mit biopsycho-sozialen Faktoren leiden, ist die multimodale Schmerztherapie. Sie erfordert ein multidisziplinäres Team aus Ärzten verschiedener Fachrichtungen, Psychotherapeuten, Bewegungstherapeuten, Sozialarbeitern und ggf. Arbeitsmarktberatern, die interdisziplinär behandeln. Diese Interdisziplinarität kann von Einzelpraxen nicht gewährleistet werden. Deshalb werden in der ambulanten Versorgung medizinische Versorgungszentren, Programme zur Integrierten Versorgung oder Netzwerke zwischen Ärzten und Therapeuten - im Optimalfall verbunden mit einer stationären Einheit - am ehesten der Forderung nach einer abgestimmten multidisziplinären Behandlung gerecht.

Man geht davon aus, dass hochchronifizierte Schmerzpatienten während des Krankheitsverlaufs eine kontinuierliche ambulante Versorgung benötigen und oft auch stationär behandelt werden müssen. Sektorenübergreifende Versorgungsstrukturen sind für die adäquate Behandlung chronischer Schmerzen somit zwingend erforderlich. Diese ermöglichen es, Termine bei unterschiedlichen Ärzten und Therapeuten zeitlich sinnvoll aufeinander abzustimmen. Zudem kann bei Bedarf ein Spezialist eines anderen Fachgebietes zur Untersuchung hinzugezogen werden. Eine enge Zusammenarbeit von Ärzten und Therapeuten über beide Sektoren hinweg erlaubt ferner eine umfassende Behandlung aus ,einem Guss", wodurch außerdem die Arzt- bzw. Therapeut-Patientenbindung gestärkt und damit „doctor hopping" vermieden werden kann. Innerhalb eines sektorenübergreifenden Behandlungsteams besteht überdies die Möglichkeit, Therapiesettings und -ziele jederzeit und auf kurzem Weg an die aktuellen Bedürfnisse des Patienten anzupassen, was wiederum hilft, Therapieabbrüche zu vermeiden.

Eine enge Kooperation von Leistungserbringern aus beiden Sektoren, verbunden mit einem guten Informationsfluss bzgl. der Krankheitsgeschichte und der Therapieinhalte bzw. -fortschritte aller aktuellen Behandlungsmodule, stellt sicher, dass alle an der Behandlung beteiligten Ärzte und Therapeuten in gleichem Maße über einen Patienten informiert sind. So können Mehrfachuntersuchungen vermieden, wertvolle Behandlungszeit geschaffen und gleichzeitig die Budgets der beteiligten Leistungserbringer geschont werden.

Sektorenübergreifende Strukturen verbessern die Behandlungsqualität für chronische Schmerzpatienten und reduzieren gleichzeitig die Kosten. Um eine wohnortnahe Versorgung zu gewährleisten, besteht vor allem in ländlichen Räumen noch ein groBer Bedarf an sektorenübergreifenden Netzwerken und Kooperationen. Vereinzelte intergrierte Versorgungsverträge mit Krankenkassen sind hier ein Tropfen auf den heißen Stein! Es müssen mehr Anreize geschaffen werden, sektorenübergreifende $\mathrm{Be}$ handlungskonzepte im Versorgungsalltag umzusetzen - zum Wohle unserer Patienten.

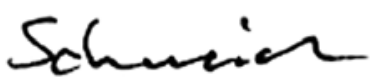

